\title{
GALECTIN-3: A NOVEL BLOOD TEST FOR THE CLASSIFICATION OF PATIENTS WITH COPD. AN OBSERVATIONAL STUDY
}

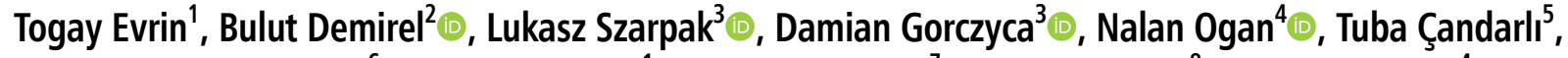

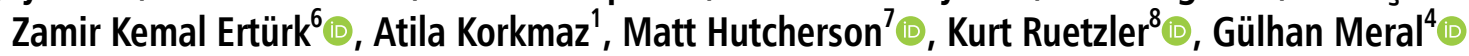 \\ 1 Ufuk University, Department of Emergency Medicine, Ankara, Turkey \\ ${ }^{2}$ Halil Sivgin Cubuk State Hospital, Department of Emergency Medicine, Ankara, Turkey \\ ${ }^{3}$ Lazarski University, Warsaw, Poland \\ ${ }^{4}$ Ufuk University, Department of Pulmonology, Ankara, Turkey \\ ${ }^{5}$ Ufuk University, Department of Biochemistry, Ankara, Turkey \\ ${ }^{6}$ Etimesgut State Hospital, Department of Emergency Medicine, Ankara, Turkey \\ ${ }^{7}$ University of Toledo College of Medicine and Life Sciences, Toledo, Ohio, United States \\ ${ }^{8}$ Department of Outcomes Research, Anaesthesiology Institute, Cleveland Clinic, Cleveland, Ohio, United States
}

\section{ABSTRACT}

INTRODUCTION: Chronic obstructive pulmonary disease (COPD) is one of the major causes of mortality and mortality affecting the whole world. In the inflammatory process, lectin is elevated and consequently, Galectin-3 expression is increased. This relation has been revealed by studies on coronary diseases. However, studies on the association of Galectin-3 with COPD and even other pulmonary diseases have been limited, although it has been studied and described on cardiologic patients. For this reason, in this study Galectin-3 levels in different stages of COPD patients were investigated and whether Galectin-3 could be a guide clinically. MATERIAL AND METHODS: This is an observational prospective study, approved by local ethic committee (30112015-12), which included three groups of patients, COPD exacerbation, COPD stable and control group, admitted to tertiary healthcare between 01.09.2016 and 01.09.2017.

RESULTS: 137 subjects were included in the study. The mean age of patients in the study was 70.6. Galectin-3 level in the group of COPD (exacerbation and stable) was significantly lower than the control group $(p<0.001)$. The Galectin-3 level was significantly lower in COPD exacerbation group than groups of stable COPD and control. And also, Galectin-3 level was significantly lower in stable COPD group than the control group ( $p$ values: $0.034,0.001$ and 0.013 , respectively). The ROC analysis for the Galectin-3 levels between the COPD patients (exacerbation and stable) and the control group is shown in Figure 2 (AUC $=0.784$ ). When the cut-off points of Galectin-3 is selected as 11.4 ; for this cut-off point, sensitivity is $83 \%$ and specificity is $71 \%$ for this cut-off point (AUC: $0.79 \% 95$ GA: $0.70-0.86 p<0.001$ ).

CONCLUSIONS: COPD is a disease with high mortality and morbidity and efforts are being made to identify its severity and exacerbations with various biomarkers. In this study, Galectin-3 levels were found to be lower in patients with stable COPD group according to the control group. In addition, galactin-3 levels were found to be lower in COPD exacerbation group according to both Stable COPD group and control group. Although a certain threshold value was found in this study, more studies are needed to determine this threshold value more precisely. However, it is clear that these data are promising.

KEY WORDS: blood test; chronic obstructive pulmonary disease; treatment; T-lymphocytes

Disaster Emerg Med J 2019; 4(3): 77-82 


\section{ABBREVIATIONS}

$A U C=$ Area under the ROC Curve

$\mathrm{CMIA}=$ Chemiluminescent microparticle

immunoassay

COPD $=$ Chronic obstructive pulmonary disease

FEV1 $=$ Forced expiratory volume in 1 second

$\mathrm{FVC}=$ Forced vital capacity

GOLD = Global Initiative for Obstructive Lung Disease

$\mathrm{PFT}=$ Pulmonary function test

$\mathrm{ROC}=$ Receiver operating characteristic

$\mathrm{WHO}=$ World Health Organization

\section{INTRODUCTION}

Chronic obstructive pulmonary disease (COPD) is one of the major causes of mortality and mortality affecting the whole world [1]. In the evaluation according to countries income estimates with the World Health Organization (WHO) data; COPD is the fifth death reason in the upper-income countries and the sixth death reason in the middle-low income countries and is responsible for $4.9-3.8 \%$ of deaths [1]. In the same report, COPD is located between the seventh and tenth row among the causes of incapacity to work [2].

According to the Global Initiative for Obstructive Lung Disease (GOLD) guidelines, COPD is a disease, which is seen with progressive irreversible airway obstruction that can affect other systems other than the lung and be prevented or treated. The COPD and its side effects can be avoided by taking measures such as non-use of cigarettes, reduction of occupational inhaler substance exposure, prevention of complications and early diagnosis of the disease. However, COPD is a treatable disease despite having a progressive feature [3]. Various classifications have been proposed to regulate the treatment of the patient and estimate the survival time but the most used classification in the world was formed according to the results of pulmonary function test (PFT), recommended by GOLD [3].

Although there are many different causes in the aetiology of COPD, the inflammation caused by inhaled particulates and gases plays a key role in the pathophysiology [4]. This situation was clearly defined in studies conducted, and now studies have focused on the relationship between the region, type and grade of inflammation and the severity of the disease. T-lymphocytes (predominantly CD8+ cells) and macrophages are present in the bronchial mucosa of COPD patients [4]. At the same time, airway obstruction correlates with the number of $\mathrm{T}$ lymphocytes [5]. However, the mechanism of this accumulation cannot yet be explained and the only known issue is that these cells and inflammation play a key role in the pathogenesis of COPD patients. As a result of this inflammation; increase in size of mucous glands in the submucosa, and hyperplasia / metaplasia and squamous metaplasia are seen in airway goblet cells [5].

Galectins are galactose-binding proteins and can enhance the proliferation of respiratory epithelial cells by limiting adhesion to the basement membrane through competition with integrin receptors for the binding of laminin in the extracellular matrix [6]. It has also been shown to play a role in the cancer mechanism. Galectin-3, which is one of ten members of the galectin family, is a $\beta$-galactosidase binding protein. Galectin-3 is also activated from $T$ lymphocytes, and it is expressed from the epithelium, and dendritic cells such as macrophages and Kupffer cells. At the same time, extracellular Galectin-3 activates monocytes/macrophages, mast cells, neutrophils and lymphocytes and regulates relations of cell-cell and cell-matrix through these activations [7].

In the inflammatory process, lectin is elevated and consequently, Galectin-3 expression is increased. This relation has been revealed by studies on coronary diseases [8]. However, studies on the association of Galectin-3 with COPD and even other pulmonary diseases have been limited, although it has been studied and described on cardiologic patients. For this reason, the authors of this study investigated Galectin-3 levels in different stages of COPD patients and whether Galectin-3 could be a guide clinically.

\section{MATERIAL AND METHODS}

This is an observational prospective study, approved by local ethic committee (30112015-12), which included three groups of patients, COPD exacerbation, COPD stable and control group, admitted to tertiary healthcare between 01.09.2016 and 01.09.2017. The patients' data were collected by the authors. The COPD exacerbation were patients admitted to emergency service; the COPD stable group, outpatients admitted to polyclinic of chest disease for COPD; the control group were healthy individuals admitted to different clinics of the hospital for control purposes. The exclusion criteria were age 
under 18 years old, active infection, cancer history, pulmonary embolism, inability to get informed of consent due to neurological and psychological reasons or tracheal intubation, absence of PFT results, and presence of active SVO. Studies exclusion criteria were defined as being under 18 years of age, active infection, presence of cancer history, absence of consent (patients with neurological and psychological reasons or with intubation), pulmonary embolism, absence of PFT result and presence of active SVO.

Demographic and clinical data as well as PFT data (FEV1, FVC, and FEV1/FVC) were registered. At hospital admission, blood sample was taken, and Architect Galectin-3 assay was performed using the chemiluminescent microparticle immunoassay (CMIA) principle for the quantitative determination of Galectin-3 in human serum. Measurements were performed on the Abbott i1000 autoanalyzer.

61 patients were included in the COPD exacerbation group. In this group; PFT results of three patients were not found, and one patient was intubated. For these reasons, 57 patients remained in this group.

49 patients were included in the group of patients with stable COPD. In this group, 2 patients were diagnosed with pneumonia, and 2 patients were diagnosed with cancer. For these reasons, 35 patients remained in this group. There were 45 people in the control group.

\section{Statistical Analyses}

Statistical analyses were performed using the IBM SPSS software version 24 . The variables were investigated using visual (histograms, probability plots) and analytical methods (Kolmogorov-Simirnov/Shapiro-Wilk's test) to determine whether they are normally distributed. Descriptive analyses were presented using median and 25 percentiles 75 percentile. The parameters were not normally distributed; "Kruskal Wallis" and "Mann-Whitney U" tests were conducted to compare these parameters, as well as to compare the ordinal variables. An overall $\% 5$ type-l error level was used to infer statistical significance. In additions, the capacity of serum galactin-3 values in predicting COPD diagnosis was analysed using ROC curve analysis. When a significant cut-off value was observed, the sensitivity and specificity were presented. While evaluating the area under the curve, a 5\% type-I error level was used to accept a statistically significant predictive value of the test variables.

\section{RESULTS}

137 subjects were included in the study as COPD exacerbations, stable COPD and control group. There were 57 patients in the COPD exacerbation group, 35 patients in the stable COPD group and 45 people in the control group. The GOLD classifications according to the PFT results of 92 patients who were in stable COPD and exacerbation group are given in Table 1.

The age data of the control group was not existing. The mean age of patients in the study was 70.6. The lowest age was 55 and the highest age was 91 . There was no statistically significant difference between the groups when the age factor was compared according to the GOLD standards $(p=0.417)$. COPD exacerbation and stable COPD groups were similar in terms of age factor $(p=0.803)$

In the control group, the patients' gender information was not identified. On the other hand, there was no significant difference between COPD exacerbation and stable COPD groups in terms of gender $(p=0.107)$. There was no significant statistical difference between aetiologic causes of COPD exacerbation, stable COPD and control groups $(p=0.143)$.

Analysis with Galectin-3 is shown in Table 2. According to this data, Galectin-3 level in the group of COPD (exacerbation and stable) was significantly lower than the control group $(p<0.001)$.

Table 1. GOLD classifications according to PFT
results of COPD patients
\begin{tabular}{|l|c|c|}
\hline & Patients & Per cent \\
\hline PFT Gold 1 & 13 & 14.1 \\
\hline PFT Gold 2 & 31 & 33.7 \\
\hline PFT Gold 3 & 28 & 30.4 \\
\hline PFT Gold 4 & 20 & 21.7 \\
\hline Total & 92 & 100 \\
\hline
\end{tabular}

\begin{tabular}{|c|c|c|c|c|}
\hline & \multicolumn{3}{|c|}{ Galectin-3 } & \multirow[b]{2}{*}{$P$ value } \\
\hline & Median & 25 Percentile & 75 Percentile & \\
\hline Control & 14.5 & 12.5 & 18.9 & \multirow{2}{*}{$<0.001^{*}$} \\
\hline COPD & 5.85 & 2.1 & 12.85 & \\
\hline
\end{tabular}


Table 3. Galectin-3 levels of COPD exacerbation, COPD stable and Control groups

\begin{tabular}{|c|c|c|c|c|c|c|}
\hline \multicolumn{5}{|c|}{ Galectin-3 } & \multicolumn{2}{|l|}{ Galectin-3 } \\
\hline & Median & 25 Percentile & 75 Percentile & $P$ value & Pairwise Comparisons & $P$ value \\
\hline COPD exacerbation & 3.80 & 1.90 & 10.7 & \multirow{3}{*}{$<0.001$} & COPD exacerbation - COPD stable & 0.034 \\
\hline COPD stable & 8.20 & 4.85 & 17.00 & & COPD exacerbation -Control & $<0.001$ \\
\hline Control & 14.50 & 12.50 & 18.90 & & COPD stable - Control & 0.013 \\
\hline
\end{tabular}

*Kruskal-Wallis Test

Independent-Samples Kruskal-Wallis Test

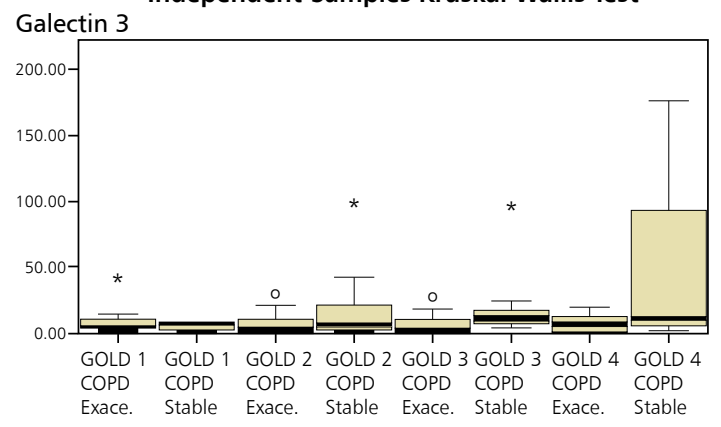

FIGURE 1. Galectin-3 values according to SFT GOLD levels of COPD exacerbation and COPD stabilized groups

Galectin-3 levels in groups of COPD exacerbation, stable COPD and control are given in Table 3. According to this table, Galectin-3 level was significantly lower in COPD exacerbation group than groups of stable COPD and control. And also, Galectin-3 level was significantly lower in stable COPD group than the control group ( $p$ values: 0.034 , 0.001 and 0.013 , respectively).

Galectin-3 levels in COPD exacerbation and stable COPD groups are given in Figure 1 according to GOLD classification. There was no significant difference between the groups according to this table $(p=0.114)$.

The ROC analysis for the Galectin-3 levels between the COPD patients (exacerbation and stable) and the control group is shown in Figure 2 $(A \cup C=0.784)$.

When the cut-off points of Galectin-3 is selected as 11.4; for this cut-off point, sensitivity is $83 \%$ and specificity is $71 \%$ for this cut-off point (AUC: $0.79 \% 95$ GA: 0.70-0.86, $p<0.001$ ).

The ROC curve analysis for the Galectin-3 level of the COPD exacerbation group and the other patients (together with COPD exacerbation and control group) is given in Figure 3. For the detection of COPD exacerbation and other conditions, when the level of Galectin-3 was selected as 9.8; for this level, sensitivity was $75 \%$ and specificity was $74 \%$ (AUC: $0.76 \% 95$ GA: 0.67-0.85, p < 0.001).

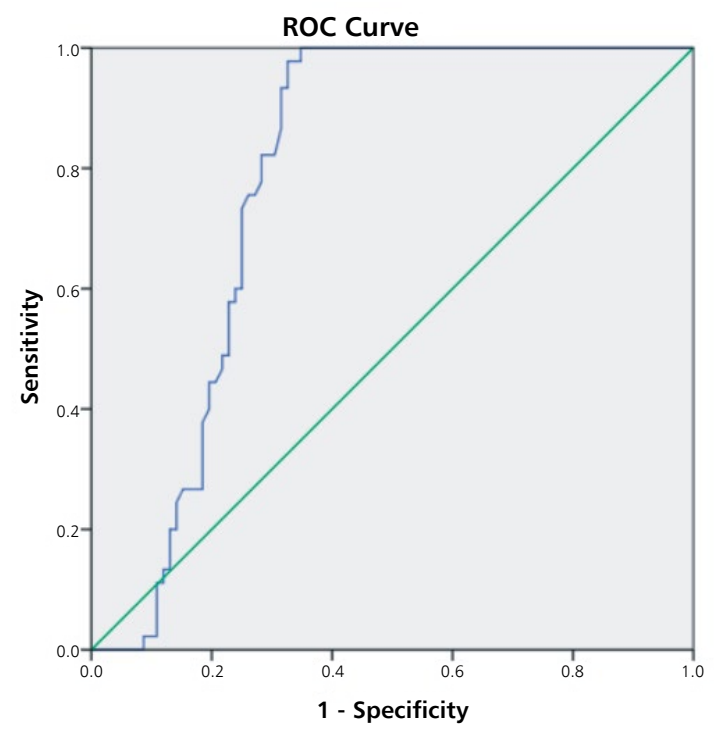

Diagonal segments are produced by ties.

FIGURE 2. ROC analysis of the Galectin-3 level between COPD patients (attack and stable) and control group

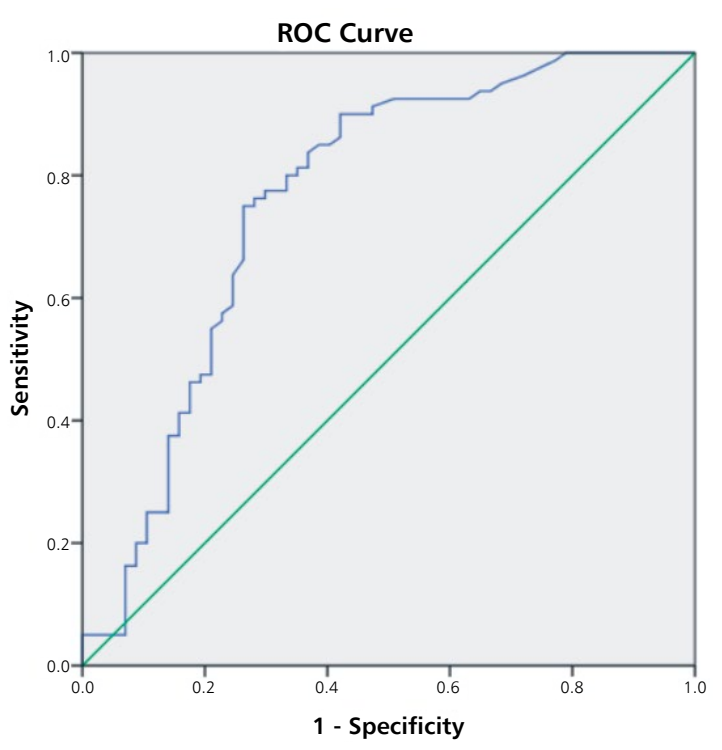

Diagonal segments are produced by ties.

FIGURE 3. ROC curve analysis for the Galectin-3 level of the COPD exacerbation group and other patients (COPD exacerbation and Control group) 


\section{DISCUSSION}

COPD is an advanced age disease due to the cumulative effect of factors that cause COPD [9]. This cumulative effect is the reason why the mean age of COPD patients is high, and this is consistent with the literature. It is also seen that there are more patients with GOLD grade 2 and 3 when the patients with COPD are classified with GOLD. When patients with COPD were separated by GOLD classification according to the PFT values, it is seen that there are more patients whose GOLD stages are 2 and 3. The reason for this may be considered that COPD patients in GOLDStage 1 are asymptomatic and take less diagnosis [10, 11]. In addition, the ratio of patients in GOLD-Stage 4 class can be thought to be relatively low due to morbid conditions associated with COPD [12]. The results of our study can be interpreted in this way.

Galectin-3 is an important element in the actin pathway of the phagocytic process and can play an important role in the production of glutathione, an intracellular antioxidant $[13,14]$. Galectin production is thought to play a role in the pathogenesis of COPD. In particular, Galectin-3 regulates apoptosis of epithelial and inflammatory cells and activation of neutrophil/macrophage [15].

Galectin-3 has been investigated in many types of cancer because of its association with cellular adhesion molecules. It has been proposed as a biomarker to predict the endpoint in different types of thyroid cancers [16]. In addition, it has been reported in relation to the prognosis, severity and endpoint of cardiac diseases (especially heart failure) in both human and rat studies [17-19].

There is a limited study of the status of Galectin-3 in COPD patients and most of them are studies with bronchoalveolar lavage at the cellular level. There is no study about the relationship between COPD and serum Galectin-3. In this study, serum Galectin-3 levels were significantly lower in COPD patients than in the Control group. And it was observed that Galectin-3 was also produced less in exacerbation group among COPD groups. However, according to the GOLD classification, there was no difference in terms of Galectin-3 levels between groups of COPD exacerbation and stable COPD.

In a study on samples taken with bronchoalveolar lavage; active smoking and non-smoking patients with COPD, and active smoking and non-smoking volunteers were included. And in that study; Galectin-3 levels were found to be lower in COPD patients both smokers and non-smokers [13]. Sim- ilarly, Galectin-3 levels were also found to be lower in patients with COPD in the presented study. But the presented study cannot be compared with that study, because the classification was not formed according to smoking status in the presented study.

The most powerful part of this study is the ROC curve analysis. When COPD patients were evaluated together with this analysis, the sensitivity and the specificity were found to be $83 \%$ and $71 \%$, respectively, for the value of 11.4 selected as a cut-off point of Galectin-3. However, the identification of the COPD exacerbation group is a priority for the authors. In the COPD exacerbation group, it was observed that sensitivity was $75 \%$ and specificity was $74 \%$ when the cut-off point of Galectin-3 was selected as 9.8. And these values can be indicated as acceptable values.

\section{CONCLUSION}

COPD is a disease with high mortality and morbidity and efforts are being made to identify its severity and exacerbations with various biomarkers. In the presented study, Galectin-3 levels were found to be lower in patients with stable COPD group according to the control group. In addition, Galactin-3 levels were found to be lower in COPD exacerbation group according to both Stable COPD group and control group. Although a certain threshold value was found in this study, more studies are required to determine this threshold value more precisely. However, it is clear that these data are promising.

Source of support: No source of financial and material support to be declared.

\section{REFERENCES}

1. Yawn BBp, Thomashaw B, Mannino DM, et al. The 2017 Update to the COPD Foundation COPD Pocket Consultant Guide. Chronic Obstr Pulm Dis. 2017; 4(3): 177-185, doi: 10.15326/jcopdf.4.3.2017.0136, indexed in Pubmed: 28848929.

2. Gold PM. The 2007 GOLD Guidelines: a comprehensive care framework. Respir Care. 2009; 54(8): 1040-1049, indexed in Pubmed: 19650945.

3. Tan WC, Bourbeau J, Aaron SD, et al. Global Initiative for Chronic Obstructive Lung Disease 2017 Classification and Lung Function Decline in Chronic Obstructive Pulmonary Disease. Am J Respir Crit Care Med. 2018; 197(5): 670-673, doi: 10.1164/rccm.201706-1154LE, indexed in Pubmed: 28858570.

4. Bourdin A, Burgel PR, Chanez $P$, et al. Recent advances in COPD: pathophysiology, respiratory physiology and clinical aspects, in- 
cluding comorbidities. Eur Respir Rev. 2009; 18(114): 198-212, doi: 10.1183/09059180.00005509, indexed in Pubmed: 20956145.

5. Fabbri L, Romagnoli M, Corbetta L, et al. Differences in Airway Inflammation in Patients with Fixed Airflow Obstruction Due to Asthma or Chronic Obstructive Pulmonary Disease. Am J Respir Crit Care Med. 2003; 167(3): 418-424, doi: 10.1164/rccm.200203-1830c.

6. Yang RY, Hsu DK, Liu FT. Expression of Galectin-3 modulates T-cell growth and apoptosis. Proc Natl Acad Sci U S A. 1996; 93(13): 67376742, doi: 10.1073/pnas.93.13.6737, indexed in Pubmed: 8692888.

7. Dumic J, Dabelic S, Flögel M. Galectin-3: an open-ended story. Biochim Biophys Acta. 2006; 1760(4): 616-635, doi: 10.1016/j. bbagen.2005.12.020, indexed in Pubmed: 16478649.

8. Hsu DK, Yang RY, Pan Z, et al. Targeted disruption of the Galectin-3 gene results in attenuated peritoneal inflammatory responses. Am J Pathol. 2000; 156(3): 1073-1083, doi: 10.1016/50002-9440(10)64975-9, indexed in Pubmed: 10702423.

9. Jarad N. Chronic obstructive pulmonary disease (COPD) and old age? Chron Respir Dis. 2011; 8(2): 143-151, doi: 10.1177/1479972311407218, indexed in Pubmed: 21596895.

10. Çolak Y, Afzal S, Nordestgaard BG, et al. Prognosis of asymptomatic and symptomatic, undiagnosed COPD in the general population in Denmark: a prospective cohort study. Lancet Respir Med. 2017; 5(5): 426-434, doi: 10.1016/S2213-2600(17)30119-4, indexed in Pubmed: 28389225.

11. Mannino DM. Does Undiagnosed Chronic Obstructive Pulmonary Disease Matter? Am J Respir Crit Care Med. 2016; 194(3): 250-252, doi: 10.1164/rccm.201602-0295ED, indexed in Pubmed: 27479056.

12. Sin DD, Anthonisen NR, Soriano JB, et al. Mortality in COPD: Role of comorbidities. Eur Respir J. 2006; 28(6): 1245-1257, doi: 10.1183/09031936.00133805, indexed in Pubmed: 17138679.
13. Mukaro VR, Bylund J, Hodge G, et al. Lectins offer new perspectives in the development of macrophage-targeted therapies for COPD/ emphysema. PLoS One. 2013; 8(2): e56147, doi: 10.1371/journal. pone.0056147, indexed in Pubmed: 23441163.

14. Dobashi K, Aihara M, Araki T, et al. Regulation of LPS induced IL-12 production by IFN-gamma and IL-4 through intracellular glutathione status in human alveolar macrophages. Clin Exp Immunol. 2001; 124(2): 290-296, doi: 10.1046/j.1365-2249.2001.01535.x, indexed in Pubmed: 11422207.

15. Rabinovich GA, Rubinstein N, Toscano MA. Role of galectins in inflammatory and immunomodulatory processes. Biochim Biophys Acta. 2002; 1572(2-3): 274-284, doi: 10.1016/s0304-4165(02)00314-8, indexed in Pubmed: 12223275.

16. Orlandi F, Saggiorato E, Pivano G, et al. Galectin-3 is a presurgical marker of human thyroid carcinoma. Cancer Res. 1998; 58(14): 3015-3020, indexed in Pubmed: 9679965.

17. Kleshchenko YY, Moody TN, Furtak VA, et al. Human Galectin-3 promotes Trypanosoma cruzi adhesion to human coronary artery smooth muscle cells. Infect Immun. 2004; 72(11): 6717-6721, doi: 10.1128/IAI.72.11.6717-6721.2004, indexed in Pubmed: 15501810.

18. van Kimmenade RR, Januzzi JL, Ellinor PT, et al. Utility of amino-terminal pro-brain natriuretic peptide, Galectin-3, and apelin for the evaluation of patients with acute heart failure. J Am Coll Cardiol. 2006; 48(6): 1217-1224, doi: 10.1016/j.jacc.2006.03.061, indexed in Pubmed: 16979009.

19. Shah RV, Chen-Tournoux AA, Picard MH, et al. Galectin-3, cardiac structure and function, and long-term mortality in patients with acutely decompensated heart failure. Eur J Heart Fail. 2010; 12(8): 826-832, doi: 10.1093/eurjhf/hfq091, indexed in Pubmed: 20525986. 